\title{
IV.1 Text als Paradigma der Kulturwissenschaft
}

\section{Semiotik als Paradigma}

Wenn man sich die Frage nach dem Ursprung des Textbegriffs innerhalb der Kulturwissenschaft stellt, fällt auf, dass jeder Versuch, Kultur als Text zu lesen, eine eingeschränkte Version des größeren Projekts der Semiotik darstellt, die Kultur als Zeichensystem zu verstehen. Ferdinand de Saussure nannte dieses Vorhaben „Semeologie“ (sémiologie) und bekräftigte, dass die Sprachwissenschaft „Musterbeispiel und Hauptvertreter der ganzen Semeologie“ (Saussure 2001, 80) werden kann, da sie sich mit Zeichen befasst, die in ihrer Arbitrarität am stärksten bezeugen, dass Bedeutung gesellschaftlichen Konventionen entspringt und den Zeichen nicht innewohnt. Doch fügt er gleich hinzu, dass „die Sprache nur ein System unter anderen ist“ (Saussure 2001, 80) und die Sprachwissenschaft nur ein Teilbereich einer umfassenderen Wissenschaft: „Man kann sich also vorstellen eine Wissenschaft, welche das Leben der Zeichen im Rahmen des sozialen Lebens untersucht; [...] wir werden sie Semeologie (von griechisch sēmeîon, ,Zeichen') nennen.“ (Saussure 2001, 19) „Die Sprachwissenschaft“, fährt er fort, ,ist nur ein Teil dieser allgemeinen Wissenschaft, die Gesetze, welche die Semeologie entdecken wird, werden auf die Sprachwissenschaft anwendbar sein, und diese letztere wird auf diese Weise zu einem ganz bestimmten Gebiet in der Gesamtheit der menschlichen Verhältnisse gehören.“ (Saussure 2001, 19)

Folgt man Saussures Argument, kommt man zum Schluss, dass die späteren Versuche, Kultur als Text zu lesen, welche im Zentrum der folgenden Ausführungen stehen, die Verhältnisse auf den Kopf stellen. War für Saussure die Sprachwissenschaft noch ein Teilbereich der Wissenschaft, welche die Kultur die „Gesamtheit der menschlichen Verhältnisse“ - aufschlüsseln helfen soll, weiten Strukturalismus und Poststrukturalismus das Anwendungsfeld der sprachlichen Analyse ungemein aus und riskieren damit, die Kultur auf Text $\mathrm{zu}$ reduzieren. Zumindest ist die Emergenz von Text als Paradigma der Kulturwissenschaft weitaus weniger Saussures Ruf nach einer Wissenschaft von den Zeichen geschuldet als der transdisziplinären Strahlkraft der von ihm ins Leben gerufenen allgemeinen Sprachwissenschaft.

Für die Entwicklung des Strukturalismus sind drei Weichenstellungen Saussures von entscheidender Bedeutung: sein Fokus auf die Sprache als gesellschaftliches System (langue) statt auf individuelle sprachliche Äußerungen 
(parole), seine Bestimmung des Werts von Zeichen als relational und differentiell und seine Analyse der Sprache mit Hilfe binärer Modelle (langue/parole, Signifikant/Signifikat, Syntagma/Paradigma). Auf diesen wegweisenden Entscheidungen baut das strukturalistische Programm letztlich auf. $\mathrm{Zu}$ nennen wären hier etwa Bestrebungen der strukturalistischen Erzähltheorie Gérard Genettes, die allen literarischen Erzählungen zugrunde liegende ,Grammatik ${ }^{\natural} \mathrm{zu}$ bestimmen (Genette 1998), Roman Jakobsons und Claude Lévi-Strauss' akribische Analyse textinterner Beziehungen in Charles Baudelaires Gedicht Les Chats (Jakobson und Lévi-Strauss 2007), Jakobsons Bestimmung der ,poetischen Funktion' als Projektion des „Prinzip[s] der Äquivalenz von der Achse der Selektion auf die Achse der Kombination“ (Jakobson 1979b, 94) und Roland Barthes' Entwurf einer mit binären Begriffspaaren (Funktionen/Indizien, Kardinalfunktionen/Katalysen, personal/apersonal) operierenden strukturalen Analyse von Erzählung (Barthes 1988a).

\section{Strukturalismus: Roland Barthes und Claude Lévi-Strauss}

Mit dem französischen Literaturwissenschaftler Barthes und dem belgischen Kulturanthropologen Lévi-Strauss sind zwei zentrale Vertreter des Strukturalismus genannt, welche linguistische Methoden der Sprachanalyse wirkungsmächtig zur Kulturanalyse erweitern. Während Ersterer die eigene, westliche Kultur einer strukturalistischen Lektüre unterzieht, wendet sich Letzterer fremden Kulturen zu.

In Mythen des Alltags (1957) widmet sich Barthes vornehmlich der französischen Kultur. In Barthes' eigener Einschätzung verfolgen seine Aufsätze zwei unterschiedliche, in den Einzelanalysen verschränkte Ziele: denaturalisierende „Ideologiekritik“ kleinbürgerlicher Vorstellungen und „semiologische Analyse“ massenkultureller Ereignisse und Artefakte, in denen sich diese Vorstellungen manifestieren (Barthes 2010a, 9). Wenn LeserInnen des 21. Jahrhunderts Barthes' metaphorisch erweiterter Gebrauch von ,Sprache“ in Wendungen wie „die Sprache der sogenannten Massenkultur“ oder "die Demontage dieser Sprache“ (Barthes 2010a, 9) kaum auffällt und seine breite Anwendung auf Phänomene wie Wrestling, Striptease, Filme und Spielsachen nicht verwundert, dann ist dies der Osmose des strukturalistischen Verständnisses von Sprache in die Alltagssprache geschuldet.

Erst wenn Barthes im Vorwort den Mythos in aller Deutlichkeit als sprachliches Phänomen bezeichnet, wird wieder gegenwärtig, dass diese sehr weite 
Auffassung von Sprache eine Geschichte hat: „Der Mythos ist eine Sprache“ (Barthes 2010a, 11). In seinem programmatischen Aufsatz Der Mythos heute präzisiert Barthes, der Mythos sei „eine Rede“ (Barthes 2010a, 251), welche von einer Vielzahl von Gegenständen und gesellschaftlichen Praktiken vermittelt werden könne: „Diese Rede ist eine Botschaft. Sie muss keine mündliche sein, sondern kann aus Schriftzeichen oder Darstellungen bestehen. Der schriftliche Diskurs, aber auch die Photographie, der Film, die Reportage, der Sport, Schauspiele, Werbung, all das kann als Träger der mythischen Rede dienen.“ (Barthes 2010a, 252) Im Folgenden weitet Barthes den Begriff der Rede noch weiter aus. Sie ist nun nicht mehr nur das, was von den Trägern vermittelt wird; die Träger selbst sind Rede: „Wir werden eine Photographie mit demselben Recht als Rede betrachten wie einen Zeitungsartikel; die Objekte selbst können Rede werden“ (Barthes 2010a, 253). Genauer gesprochen, die Träger - das Cover von ParisMatch, auf dem ein schwarzer französischer Soldat stolz militärisch grüßt - sind Rede in einem semiologischen Sinne: Signifikanten, deren Signifikat ein mythischer Begriff ist - in diesem Beispiel die „französisch[e] Imperialität“ (Barthes 2010a, 263).

Gegen Ende von Der Mythos heute benennt Barthes schließlich zwei Gegenspieler der mythischen Rede: den kulturkritischen Mythologen, der sich der Semiologie bedient, um die Mythen des Alltags zu entziffern und als Mythen kenntlich zu machen (Barthes 2010a, 312-316) und die selbstbezügliche poetische Rede zeitgenössischer nichtmimetischer Dichtung, welche „die Verbindung zwischen Signifikant und Signifikat bis zur Grenze des Möglichen [lockert]“ und „vorgibt, sich in ein System von Essenzen zurückzuziehen“ (Barthes 2010a, 283284). Barthes’ Überlegungen zur Widerständigkeit selbstreferentieller Dichtung reihen sich in eine Tradition des Nachdenkens über die Literatur ein, welche gerade in der Andersartigkeit literarischer Sprache, in ihrer Poetizität, kulturkritisches Potential sieht. Theodor W. Adorno wird genau das in einem berühmten Paradox auf den Punkt bringen: „Asozialität wird zur sozialen Legitimation von Kunst.“ (Adorno 1970, 348)

Das Adjektiv ,elementar‘ im Titel von Lévi-Strauss’ 1949 erschienener Schrift Die elementaren Strukturen der Verwandtschaft bezieht sich zunächst auf ein System der Verwandtschaftsstruktur, welches in vorindustriellen Gemeinschaften vorherrscht und starren Regeln bezüglich der Wahl des Gatten unterworfen ist: „Unter ,elementaren Strukturen der Verwandtschaft“ verstehe ich [...] Systeme, die zwar alle Mitglieder der Gruppe als Verwandte definieren, diese jedoch in zwei Gattungen unterteilen: mögliche Gatten und verbotene Gatten." (Lévi-Strauss 1993, 15) Hier zeigt sich nicht nur eine grundlegende Gemeinsamkeit zwischen Lévi-Strauss' und Barthes' strukturalistischen Zugriffen auf Kultur - die binäre Kodierung der Analysekategorien -, sondern es zeigen sich auch zwei wesentli- 
che Unterschiede zwischen einem literaturwissenschaftlichen und einem kulturanthropologischen Strukturalismus. Der eine wurde bereits benannt: Barthes widmet sich der eigenen Kultur, Lévi-Strauss hingegen fremden Kulturen (im Wesentlichen den östlichen und südlichen Teilen Asiens sowie Australien). Der zweite Unterschied ist, dass sich die Kulturanthropologie der ersten Hälfte des 20. Jahrhunderts Kulturen zuwendet, die sie als einfacher, in sich geschlossener und ,primitiver ' als ihre eigene Kultur versteht. Für Lévi-Strauss hat die Beschäftigung mit ,elementareren' Gesellschaftssystemen einen heuristischen Mehrwert, da man das strukturalistische Modell anhand einfacher Gegenstände entwickeln kann: „Streng genommen ist die vorliegende Arbeit also eine Einführung in eine allgemeine Theorie der Verwandtschaftssysteme. Denn nach dieser Studie über die elementaren Strukturen bedarf es einer anderen über die komplexen Strukturen.“ (Lévi-Strauss 1993, 16) Lévi-Strauss lässt offen, ob es für die Analyse komplexerer Systeme anderer Modelle bedarf, doch es darf bezweifelt werden, dass dies für ihn ein genuines Forschungsdesiderat ist, denn mit der Ausarbeitung einer „allgemeine[n] Theorie der Verwandtschaftssysteme“ ist das strukturalistische Ziel bereits erreicht. Bezeichnenderweise gab Lévi-Strauss sein Vorhaben, seine Analysen in einer zweiten Monographie auf ,komplexere‘ Gesellschaftssysteme auszuweiten, auf. Es zeigt sich hier eine zweite mögliche Bedeutung des Adjektivs ,elementar' im Titel seines Buchs: Es geht ihm auch in einem strukturalistischen Sinne um Elementares, das heißt, um grundlegende, universelle Strukturen einer langue der Verwandtschaft. In der kulturanthropologischen Spielart des Strukturalismus ist diese Suche nach elementar-universellen kulturellen Grammatiken untrennbar mit der Fokussierung auf als elementar-einfach verstandene Gemeinschaften verbunden.

Wie bei Barthes sind es auch bei Lévi-Strauss die binären Kodierungen, welche am beredtesten Zeugnis von seinem strukturalistischen Zugriff ablegen: elementare/komplexe Strukturen der Verwandtschaft, mögliche Gatten/verbotene Gatten, Exogamie/Endogamie, Natur/Kultur. In Lévi-Strauss' wiederholter Verwendung des Begriffs ,Dichotomie' zeigt sich ein weiteres Wesensmerkmal des Strukturalismus, der sich die Frage stellen muss, inwieweit binäre Unterscheidungen bloß ein heuristisches Werkzeug der Wissenschaft sind oder die Untersuchungsobjekte selbst strukturieren. Lévi-Strauss' Antwort ist differenziert: Manchmal gehe der Soziologe so vor wie der Genetiker, bei dem „eine strenge Übereinstimmung zwischen dem analytischen Verfahren und seinem Gegenstand“ (Lévi-Strauss 1993, 180) bestehe, manchmal so wie der Mathematiker, bei dem dies nicht der Fall sei. Die allgemeine Stoßrichtung ist jedoch klar: Lévi-Strauss erhebt das menschliche Vermögen, die Welt binär zu kodieren, gar zu einem Wesensmerkmal des Kulturzustands (Lévi-Strauss 1993, 215). Mehr noch, man „wird vielleicht einräumen müssen, dass die Dualität, die Alternanz, 
der Gegensatz und die Symmetrie [...] nicht so sehr Phänomene sind, die es zu erklären gilt, als vielmehr die fundamentalen und unmittelbaren Gegebenheiten der geistigen und sozialen Realität“ (Lévi-Strauss 1993, 215). Die Kultur ist hier nicht in dem Sinne Sprache, dass sie wie ein Text gelesen werden kann, sondern weil sie selbst, sowohl in ihrer Manifestation im individuellen Bewusstsein wie auch in der empirischen Wirklichkeit, wie eine Sprache strukturiert ist, nämlich dichotom und relational. Es ist deshalb nur folgerichtig, dass sich bei Lévi-Strauss Natur und Kultur nicht nur im Unvermögen/Vermögen der binären Kodierung, sondern auch in der Absenz/Präsenz von Sprache unterscheiden: „Mein Vorschlag war, dass man sich bei der Festlegung der Demarkationslinie zwischen beiden Ordnungen von der Anwesenheit oder Abwesenheit der artikulierten Sprache leiten läßt.“ (Lévi-Strauss 1993, 23)

Während die zwei prominentesten Vertreter der strukturalistischen Linguistik - Jakobson und Saussure - in Die elementaren Strukturen der Verwandtschaft nur in den Danksagungen erscheinen (Saussure gar nur über seinen Sohn Raymond), reflektiert Lévi-Strauss in einer Reihe von Aufsätzen aus den 1940er und 1950er Jahren eingehender über das Verhältnis von Sprache und Kultur sowie jenes von Sprachwissenschaft und Sozialwissenschaft. In Die Strukturanalyse in der Sprachwissenschaft und in der Anthropologie (1945, Lévi-Strauss 1967b), Sprache und Gesellschaft (1951, Lévi-Strauss 1967c) und Sprachwissenschaft und Anthropologie (1952, Lévi-Strauss 1967d) gesteht er der Sprachwissenschaft eine Führungsrolle zu und verlangt, dass sich die Anthropologie ihrer Analysekategorien und Methoden bediene. Im Zentrum stehen drei Aneignungen: die Analyse des Untersuchungsgegenstands in seine kleinsten Einheiten; die grundlegende Einsicht, dass diesen Einheiten nur in ihren wechselseitigen Beziehungen innerhalb eines Systems Bedeutung zuwächst, und die Suche nach einer universalen, binär kodierten Grammatik von Mythen und Kulturen. In seinem vielzitierten Aufsatz Die Struktur der Mythen (1955) entwickelt er schließlich seine Methode der Mythenanalyse explizit auf der Grundlage von Saussures und Émile Benvenistes Sprachtheorien und zerlegt den Mythos in Analogie zur Aufteilung der Sprache in Phoneme, Morpheme und Semanteme in Mytheme, welche die komplexeste der genannten Einheiten ist. Anders als Saussure und Barthes analysiert Lévi-Strauss nicht Beziehungen zwischen einzelnen Einheiten, sondern Relationen zwischen „Beziehungsbündel[n]“ (Lévi-Strauss 1967a, 232), womit die Beziehungen zwischen allen Elementen auf der paradigmatischen Achse gemeint sind. Dennoch ist für Lévi-Strauss wie für Barthes der Mythos eine Sprache, nicht nur weil er sich sprachlich artikuliert, sondern auch weil er wie eine Sprache funktioniert und strukturiert ist. 


\section{Derridas Schriftbegriff, Foucaults Diskursanalyse, Butlers Genderbegriff}

Für Barthes und Lévi-Strauss ist letztlich noch nicht in erster Linie der Text, sondern die Sprache das Paradigma der Kultur- und Sozialwissenschaft. Barthes spricht vom Mythos gemäß der ursprünglichen Bedeutung des Wortes $\mu$ ṽ 0 o (mythos) gar explizit als ,Rede‘. Erst Jacques Derridas dezidierte Abkehr vom Phonozentrismus bringt eine zweite Engführung mit sich. Wir erinnern uns: Die strukturalistische Entscheidung, Kultur als Sprache zu lesen, ist bereits eine Eingrenzung des Saussure'schen Projekts der Semeologie, da es ein Zeichensystem unter vielen zum Modell aller Zeichensysteme erklärt. Die zweite Engführung, die Erhebung von Text zum Paradigma der Kulturwissenschaft, findet erst in dem Moment statt, in dem Derrida die Schrift und nicht die Stimme als akkurateres Modell für die Sprache an sich bestimmt. Nach Derrida ist es die Kontextunabhängigkeit geschriebener Sprache, die Tatsache, dass sie anders als die Rede von spezifischen Kontexten der Produktion und Rezeption loslösbar und jenseits dieser zu verstehen ist, der primäre Grund, wieso Text und Schrift die besseren Modelle für das Verständnis von Sprache liefern. Wert und Bedeutung sind sprachlichen Zeichen nicht inhärent, sondern kommen ihnen innerhalb des Systems der Sprache zu. Sie gehorchen einer Logik der Iterabilität, der Wiederholbarkeit mit einer Differenz, welche unabhängig von spezifischen Manifestationen oder Bezugnahmen auf eine außersprachliche Welt gilt. So kann etwa ein literarischer Text in gänzlich anderen geographischen Räumen gelesen und verstanden werden, als er geschrieben wurde und hunderte von Jahren nach dem Tod seines Autors oder seiner Autorin. Diese Kontextunabhängigkeit und Zitierbarkeit der Zeichen ist ein wesentliches Merkmal der Sprache im Allgemeinen. Sprache ist demnach Urschrift, ,allgemeiner Text“ (Derrida 1988a, 93, 120). Alles ist Text.

Derridas wohl berühmteste Bemerkung, „Ein Text-Äußeres gibt es nicht“ (Derrida 1983, 274), bezieht sich allerdings in erster Linie auf die der Sprache inhärente Logik der différance - ein Begriff, der die bereits von Saussure geäußerte Einsicht, dass es „in der Sprache [...] nur Verschiedenheiten ohne positive Einzelglieder“ (Saussure 2001, 143) gibt, in dem Sinne radikalisiert, dass er nicht nur die relationale und differentielle Natur der Zeichen benennt, sondern auch die Prozessualität und Selbstbezüglichkeit des Sprachspiels. Signifikanten verweisen immer nur auf andere Signifikanten, nie aber auf Signifikate (geschweige denn auf empirische Objekte), und Bedeutung wird stets aufgeschoben. In Derridas eigenen Worten: „Es hat immer nur Supplemente, substitutive Bedeutungen gegeben, die ihrerseits nur aus einer Kette von differentiellen Verweisen hervorgehen konnten, zu welchen das ,Wirkliche' nur hinzukam, sich lediglich 
anfügte, wenn es - ausgehend von einer Spur und einem Ergänzungszeichen usw. - Bedeutung erlangte.“ (Derrida 1983, 274-275) Mit Derrida gelangen wir zu einem spezifischen Verständnis von Kultur als Text als unabgeschlossen, supplementär und spielhaft. Dies gilt es im Auge zu behalten, wenn wir auf den Textbegriff, den die Writing-Culture-Debatte in die Kulturanthropologie einführte, zu sprechen kommen.

Zunächst aber sei daran erinnert, dass Derridas poststrukturalistisches Programm in kritischer Auseinandersetzung mit der Kulturanthropologie entstand. Es war sein am 21. Oktober 1966 an der Johns Hopkins University gehaltener Vortrag Die Struktur, das Zeichen und das Spiel im Diskurs der Wissenschaften vom Menschen, welcher die Dekonstruktion in die Vereinigten Staaten einführte. Derrida hielt den Vortrag im Rahmen des Kolloquiums The Language of Criticism and the Sciences of Man, welches unter anderem darauf angelegt war, das Werk eines der Redner, Lévi-Strauss, zu würdigen. Dies hilft, die Sprengkraft von Derridas Vortrag zu erklären, denn Lévi-Strauss' Überlegungen zum Inzestverbot sind der Ausgangspunkt seiner Kritik an der binären Kodierung strukturalistischen Denkens. Für Lévi-Strauss ist das Inzestverbot eine Anomalie und kommt „nahezu“ einem „Skandal“ (Lévi-Strauss 1993, 52) gleich, weil es sowohl universell gilt, als auch kulturell spezifischen Normen und Regeln unterworfen ist und damit an den zwei inkommensurablen Ordnungen der Natur und Kultur partizipiert. Für Derrida hingegen ist das Inzestverbot die Aporie, in der das Scheitern des binären Denkens in Gegensatzpaaren wie Natur/Kultur in Erscheinung tritt. Bereits Lévi-Strauss verstehe, so Derrida, dass der Mythos letztlich kein Zentrum, keinen Ursprung und keine Autorschaft hat, die die binäre Ordnung garantieren könnten (Derrida 2000a, 432-433), doch in seiner Beschäftigung mit fremden, ,elementareren' Kulturen verleihe er einem nostalgischen Begehren nach Unmittelbarkeit und Präsenz Ausdruck, dies am deutlichsten in Traurige Tropen (1955) (Derrida 2000a, 440-441). Wenn man, wie Derrida, versucht, sich von einer ,Metaphysik der Präsenz' vollständig zu lösen, beginnt man zu verstehen, dass es in der Absenz eines Zentrums nichts mehr gibt, was das freie Spiel der Signifikanten eingrenzt: „Mit diesem Augenblick bemächtigt sich die Sprache des universellen Problemfeldes. Es ist dies auch der Augenblick, da infolge der Abwesenheit eines Zentrums oder eines Ursprungs alles zum Diskurs wird [...], das heißt zum System, in dem das zentrale, originäre oder transzendentale Signifikat niemals absolut, außerhalb eines Systems von Differenzen, präsent ist. Die Abwesenheit eines transzendentalen Signifikats erweitert das Feld und das Spiel des Bezeichnens ins Unendliche.“ (Derrida 2000a, 424)

Wie Derrida in der Grammatologie weiter ausführt, ist das Verständnis von Text, welches diesem Denken der Dezentrierung und Substitution verhaftet ist, im Gegensatz zur Idee des ,Buchs‘ zu verstehen, das heißt als unabgeschlossen, 
unbestimmt und radikal offen (Derrida 1983, 16-48). Mit diesem Textverständnis erhält die Kulturwissenschaft ein heuristisches Werkzeug, mit dem sich Kultur als dynamisches System endloser Semiose verstehen lässt. Gleichzeitig verweist Derridas Nennung des Diskursbegriffs auf Foucaults parallel entstehende Diskursanalyse und ihren viel dezidierteren Fokus auf den performativen Charakter von Sprache und deren Rolle in der Herstellung von Wissen und Reproduktion von Macht.

Während Derrida die befreienden Momente eines Denkens jenseits transzendentaler Signifikate wie Bewusstsein, Mensch oder Gott hervorhebt und den Spielcharakter des nicht abschließbaren Zeichentauschs betont, richtet Foucault sein Augenmerk auf Festsetzungen durch Aussageformationen und sieht seine Aufgabe darin, „nicht - nicht mehr - die Diskurse als Gesamtheiten von Zeichen (von bedeutungstragenden Elementen, die auf Inhalte oder Repräsentationen verweisen), sondern als Praktiken zu behandeln, die systematisch die Gegenstände bilden, von denen sie sprechen“ (Foucault 1981, 74). In seiner Fokussierung auf die Macht- und Subjekteffekte von Sprache und Denken bricht Foucault mit dem modernen, humanistisch-aufgeklärten Verständnis vom Menschen als selbstbestimmtes, autonomes Individuum und zeigt auf, wie Subjekte diskursiv erschaffen werden. So zeichnet er etwa im ersten Band seiner Geschichte der Sexualität (1976) die Produktion des ,Homosexuellen“ durch psychiatrische, juristische und literarische Praktiken, Untersuchungen, Schriften und Reden des 19. Jahrhunderts nach (Foucault 1998, 58).

Mit Judith Butler, deren Theorie der Performativität sowohl auf Foucaults Diskursanalyse aufbaut wie auch auf Derridas kritischer Auseinandersetzung mit John L. Austins Unterscheidung zwischen normalen und parasitären performativen Sprechakten (Derrida 1988b), rücken die diskursive Konstruktion von Geschlecht und die Einschreibung der Diskurse in Körper ins Zentrum des Interesses. Uwe Wirth spricht hier von den „Verkörperungsbedingungen“ (Wirth 2002a, 42) des Performativen. In Butlers eigenen Worten: „Hinter den Äußerungen der Geschlechtsidentität (gender) liegt keine geschlechtlich bestimmte Identität (gender identity). Vielmehr wird diese Identität gerade performativ durch die ,Äußerungen“ konstituiert, die angeblich ihr Resultat sind.“ (Butler 1991, 49) Stärker als Foucault betont Butler jedoch die Handlungsfreiräume, welche ein antiessentialistischer Begriff von Identität sichtbar macht: „Als öffentliche Handlung und performativer Akt ist die Geschlechterzugehörigkeit keine radikale Wahl und kein radikales Projekt, das auf eine bloß individuelle Entscheidung zurückgeht, aber ebenso wenig wird es dem Individuum aufgezwungen oder eingeschrieben. [...] [D]er geschlechtsspezifische Körper [setzt] seine Rolle in einem kulturell beschränkten Körperraum um und inszeniert Interpretationen innerhalb der Grenzen bereits gegebener Anweisungen.“ (Butler 2002, 313) Das 
Subjekt konstituiert sich in einem Wechselspiel von struktureller Determination (Performativität) und einer Selbstinszenierung (Performanz), die immer schon gesellschaftlich vorgeformt, nie aber völlig determiniert ist. Die Einsicht, dass Körper und Subjekte nicht biologisch bestimmt, sondern diskursiv konstituiert sind, öffnet den Blick für die Möglichkeit des iterativen Zitats, der Wiederholung tradierter Verhaltensmuster und Geschlechternormen mit einer Freiräume öffnenden Differenz. Darin liegt „das politische Versprechen der performativen Äußerung“ (Butler 1997, 252).

Mit den Begriffen der Schrift (Derrida), des Diskurses (Foucault) und der Performativität/Performanz (Butler) erhält die Literatur- und Kulturwissenschaft drei poststrukturalistische Verständnisse von Text, welche das strukturalistische Projekt einer Untersuchung der Sprache jenseits der Referentialität weiterführen und in entscheidender Weise radikalisieren. ,Text‘ wird hier zur Chiffre für ein Sprachverständnis, welches die Sprache nicht nur von ihrem realweltlichen Verweischarakter löst, sondern auch von jeglicher Verankerung in metaphysischen Präsenzen wie Gott, Mensch oder Wahrheit. Letztere sind nun als Diskurseffekte zu verstehen, nicht als Zentren, die außerhalb der Sprache liegen und sie fundieren. Damit rückt sowohl ein befreiendes Moment in den Blick - Sprache als Sprachspiel - als auch die subjekt- und objektkonstituierende Macht von Sprache. Unter Derridas, Foucaults und Butlers Einfluss wenden sich viele Literatur- und KulturwissenschaftlerInnen von einem strukturalistischen Verständnis von Texten als geschlossene, weitgehend statische Systeme ab und betonen deren Offenheit, Prozessualität und performative Kraft.

\section{Writing Culture und New Historicism}

Wenn man bedenkt, dass eine der Initialzündungen des poststrukturalistischen Programms - Derridas Vortrag an der Johns Hopkins University - auf einer Kritik an Lévi-Strauss fußt, dann überrascht es nicht, dass dieses Programm weitreichende Rückwirkungen auf die Theoriebildung in der Kulturanthropologie hatte. Die Writing-Culture-Debatte der 1980er und 1990er Jahre nimmt die poststrukturalistische Einsicht in die wirklichkeitskonstituierende Kraft von Sprache auf und richtet ihr Augenmerk auf die Ethnographie als Schreibpraxis. Die in James Cliffords und George E. Marcus' wegweisendem Band Writing Culture: The Poetics and Politics of Ethnography (1986) versammelten Aufsätze fokussieren auf die hierarchischen Machtgefüge, welche in der Verschriftlichung fremder Kulturen zum Ausdruck gebracht und konsolidiert werden. Die ethnographische Praxis - das Schreiben, Interpretieren und Übersetzen fremder Kulturen in Texte - ist stets 
in eine Kolonialgeschichte eingebettet und schreibt diese fort. Die Mehrzahl der BeiträgerInnen in Writing Culture, unter anderem Mary Louise Pratt (2011) und James Clifford (2011), unterziehen die rhetorischen und quasiliterarischen Verfahrensweisen, mit denen ethnographische Autorität erzeugt wird, einer kritischen Analyse; andere, darunter Stephen A. Tyler (2011) und Michael M. Fischer (2011), besprechen neuere, experimentelle, nichtrealistische Formen des ethnographischen Schreibens, welche einen dialogischeren, selbstreflexiveren oder im engeren Sinne literarischen Umgang mit nichtwestlichen Kulturen praktizieren. In den Blick gerät dabei sowohl, ,wie in die Wahrnehmung von Natur und Landschaft Geschichte(n), Mythen, Legenden und Erinnerungen gleichsam eingeschrieben sind“ (Bachmann-Medick 2004, 7), als auch, wie Einschreibungen in Kultur und Verschriftlichungen von Kultur epistemologische Macht produzieren.

Ein Kulturanthropologe, der oft mit der Writing-Culture-Debatte assoziiert wird, ist Clifford Geertz. Er wird gerne als Vorläufer dieser Debatte gesehen, doch sein Fokus ist ein anderer. Geertz' Augenmerk gilt weniger der Übersetzung fremder Kulturen in ethnographische Prosa denn der textuellen Verfasstheit des „selbstgesponnene[n] Bedeutungsgewebe[s]“ (Geertz 2003a, 9) namens ,Kultur“. So schreibt er in ,Deep Play“: Bemerkungen zum balinesischen Hahnenkampf (1972): „Die Kultur eines Volkes besteht aus einem Ensemble von Texten, die ihrerseits wieder Ensembles sind, und der Ethnologe bemüht sich, sie über die Schultern derjenigen, für die sie eigentlich gedacht sind, zu lesen.“(Geertz 2003a, 259) Auch hier, in Geertz' Entschluss, die balinesische Kultur als Ansammlung von Texten zu lesen, zeigt sich das (post-)strukturalistische Erbe. Allerdings ist das Verständnis von Text, welches Geertz' interpretativer Kulturanthropologie zugrunde liegt, ein gänzlich anderes als jenes von Lévi-Strauss, Derrida oder Butler. Geertz vergleicht den balinesischen Hahnenkampf mit Shakespeare’schen Tragödien und schlägt folgerichtig vor, ihn wie ein literarisches Werk $\mathrm{zu}$ interpretieren. Ein solcher Zugang mache deutlich, dass es hier um eine von den Erfordernissen des Alltags losgelöste Verhandlung der großen Themen der balinesischen Kultur gehe - „Tod, Männlichkeit, Wut, Stolz, Verlust, Gnade und Glück“ (Geertz 2003a, 246) - und dem Ethos dieser Kultur und ihrer kollektiven Gefühlsstruktur Ausdruck verliehen werde (Geertz 2003a, 254). Geertz interpretiert den Hahnenkampf und sieht ihn zugleich als den Ort, an dem sich die balinesische Kultur selbst deutet. Bei aller Affinität zwischen Geertz' Verständnis von Kultur als Text und (post-)strukturalistischen Theoremen liegt seinem Willen, fremde Kulturen zu interpretieren, ein traditionelles Verständnis von Literatur und Interpretation zugrunde, welches weder mit Derridas Konzeptualisierung von Text als freies Spiel der Signifikanten noch mit dessen antihermeneutischem Gestus oder mit Foucaults und Butlers Fokussierung auf den Macht-Wissen-Nexus in Einklang zu bringen ist. Bezeichnenderweise unterzieht Vincent Crapanzano in seinem Beitrag zum Writing-Culture-Band 
Geertz' Selbstermächtigung als Meisterinterpret einer Kultur, deren Repräsentanten kaum zu Wort kommen (denn deren Kultur wird „über ihre Schulter“ gelesen), einer harschen Kritik (Crapanzano 2011, 68-76).

Trotz dieser Divergenzen hatte Geertz' Werk im Allgemeinen und sein Hahnenkampf-Aufsatz im Besonderen beträchtlichen Einfluss auf just jene Spielart der Literaturtheorie, die den Einzug des poststrukturalistischen Denkens in die Literaturgeschichtsschreibung markiert: den New Historicism. Im Rahmen des vorliegenden Handbuchs zu Poetizität/Poetik ist Stephen Greenblatts Skizze einer ,Poetik der Kultur‘ von besonderem Interesse. Greenblatts Überzeugung, dass vergangene Kulturen nicht wie irgendein Text gelesen werden sollen, sondern wie ein literarischer Text, knüpft an Geertz' Bestreben an, ,dichte Beschreibungen“ im Wesentlichen mikroskopische Interpretationen mit den Mitteln des close reading - einzelner kultureller Ausdrucksformen anzufertigen (Geertz 2003b). Auch für Greenblatt ist „der soziale Diskurs schon mit ästhetischer Kraft geladen“ (Greenblatt 1991, 120); auch er verfolgt Geertz' „Ziel einer Genauigkeit der Einzelbeschreibungen“, welches sich klar von „den verallgemeinernden Abstraktionen des Strukturalismus“ (Bachmann-Medick 2004, 25) absetzt; und auch er analysiert die „sozial[e] Formierungskraft kultureller Selbstauslegung, wie es Geertz am balinesischen Hahnenkampf entfaltet hat“ (Bachmann-Medick 2004, 45).

Greenblatt begreift Kultur als prozessuales Feld, in dem Texte aller Art und gesellschaftliche Praktiken dynamisch zirkulieren, verhandelt und getauscht werden. Eine ,Poetik der Kultur‘ analysiert, ähnlich wie Geertz, kulturelle Praktiken mit den Mitteln der Literaturwissenschaft und Rhetorik, wendet sich aber von einem rein textuellen Paradigma ab, indem sie die materiellen, ökonomischen und politischen Bedingungen des Zeichentauschs stets mitreflektiert. Damit schließt das Projekt der Kulturpoetik an die noch dezidierter kulturpolitisch akzentuierte Forschung innerhalb der postkolonialen Studien an (BachmannMedick 2004, 37-44). Zudem betont die ,Poetik der Kultur‘ sehr viel stärker als Geertz, dass Kunst und Gesellschaft keine getrennten Sphären, sondern durch eine „beunruhigende Zirkulation von Materialien und Diskursen“ (Greenblatt 1991, 121) untrennbar miteinander verbunden sind.

Wie in der zehnten Fußnote von Grundzüge einer Poetik der Kultur ersichtlich wird, leiht sich der Kulturpoetiker Greenblatt (1991) den Begriff der ,Poetik‘ allerdings nicht von Geertz, sondern vom Kultursemiotiker Jurij M. Lotman, welcher in Die Poetik des Alltagsverhaltens in der russischen Kultur des 18. Jahrhunderts (Lotman 1985) von der Formierung von Individuen im Wechselspiel von Text und Kultur schreibt. Lotmans Augenmerk gilt der Figur des Autors, welche im Zwischenraum von Kunst (das in den literarischen Text eingeschriebene Bild des Dichters) und Leben (der empirische Autor) in Prozessen der Theatralisierung, Maskierung und des Rollenspiels entsteht. Damit antizipiert Lotman Greenblatts 
sehr viel stärker an Machtfragen interessierte Überlegungen zum self-fashioning von (Renaissance-)Subjekten im Spannungsfeld von Handlungsermächtigung und struktureller Bestimmtheit (Schahadat 2012). In Greenblatts Modell konstituieren sich Subjekte im Rahmen der Zirkulation von Zeichen, Texten und soziokulturellen Energien (Greenblatt 1980). Lotmans strukturalistisches Verständnis von Kultur und Identität wird von poststrukturalistischer Warte aus neu gedeutet.

Am deutlichsten hält der poststrukturalistische Textbegriff allerdings in den Arbeiten eines im deutschsprachigen Bereich weit weniger breit rezipierten New Historicist Einzug. Louis A. Montroses Charakterisierung des New Historicism als eine literatur- und kulturwissenschaftliche Schule, welche sich sowohl der „Historizität von Texten“ als auch der „Textualität von Historie“ (Montrose 1989, 20; Übersetzung Ph. Sch., Hervorhebungen im Original) widmet, betont, im zweiten Teil der Doppelformel und mit Rückgriff auf Hayden Whites Analyse der narrativen und figürlichen Verfasstheit der Geschichtsschreibung, die Vermitteltheit jeglichen Zugriffs auf die Vergangenheit. In den Blick gerät dabei nicht nur die unhintergehbare textuelle Verfass theit von Geschichte, sondern auch die Einsicht, dass die Texte der Vergangenheit, welche HistorikerInnen und LiteraturwissenschaftlerInnen als Quellen oder Primärtexte bestimmen, selbst Interpretationen vergangener Welten sind und nur einen Bruchteil der schriftlichen Zeugnisse darstellen, die produziert und erhalten wurden. Zudem konstatiert Montrose, dass diese Quellen und Primärtexte im Rahmen der (Literatur-)Geschichtsschreibung weiteren Selektions- und Interpretationsvorgängen unterzogen werden. Auf all diesen Vermittlungsstufen spielen ,gesellschaftliche Prozesse der Erhaltung und Tilgung“ (Montrose 1989, 20; Übersetzung Ph. Sch.) eine zentrale Rolle, deren Einbettung in den Macht-Wissen-Nexus stets mitgedacht werden muss. Schließlich hebt Montrose hervor, dass literarische Texte nicht nur „gesellschaftlich produziert“, sondern auch „gesellschaftlich produktiv“ sind, nämlich in dem Sinne, dass „Schreiben und Lesen immer historisch und gesellschaftlich determinierte Ereignisse sind, welche von geschlechtsspezifischen Individuen und Kollektiven in der Welt vollzogen werden und auf die Welt einwirken " (performed in the world and upon the world, Montrose 1989, 23; Übersetzung Ph. Sch., Hervorhebungen im Original).

\section{Kanonpolitik des New Historicism}

New Historicists wie Greenblatt und Montrose gehen davon aus, dass Texte die kulturelle Wirklichkeit nicht primär reflektieren oder ausdrücken, sondern herstellen: Sie sind integrale Bestandteile gesellschaftlicher Debatten, formen sie 
und produzieren Subjektpositionen. Auch in dieser Betonung der performativen Kraft von Texten und von Identität als Performanz gehen die New Historicists mit poststrukturalistischen TheoretikerInnen wie Butler einig. Ihr Textverständnis hat jedoch sehr viel unmittelbarere Auswirkungen auf die literaturwissenschaftliche Praxis. In ihrem Bestreben, die Performativität und (bei Greenblatt) die ästhetische Kraft aller Texte zu ergründen und den Kanon der Literatur zu öffnen, nivellieren die New Historicists bewusst die Unterschiede zwischen literarischen und nichtliterarischen Texten. Bei ihrem historischen Zugriff geht es ihnen nicht darum, literarische Texte in ihre historischen Kontexte einzubetten, sondern Kultur als das Produkt einer Vielzahl von Ko-Texten zu verstehen. So stellt Greenblatt in Invisible Bullets: Renaissance Authority and Its Subversion (1981b) Thomas Harriots kolonialen Reisebericht A Brief and True Report of the New Found Land of Virginia (1588) gleichberechtigt neben Shakespeares Historiendrama 1 Henry IV (1597) und liest beide als homologe Inszenierungen der simultanen Produktion und Eindämmung von Subversion durch mächtige (koloniale und königliche) gesellschaftliche Akteure. Reiseberichte, Gerichtsakten, politische Pamphlete und Fibeln sind für New Historicists weit mehr als Zeugnisse der historischen Wirklichkeit, auf die literarische Texte verweisen; sie haben, wie literarische Texte selbst, wirklichkeitskonstituierende Kraft.

Diese Nivellierung der Diskurse hat ein befreiendes Moment, öffnet sie doch den literarischen Kanon für Texte sozialer Minderheiten, deren schriftliche Produktion innerhalb der Literaturwissenschaft lange wenig Beachtung fand, unter anderem deshalb, weil sie nicht oder nur teils gängigen Vorstellungen des Literarischen entsprachen. Diese Öffnung des Kanons ist insbesondere auch dem New Historicism zu verdanken, welcher bis heute das dominante Theorieparadigma innerhalb der amerikanistischen Literatur- und Kulturwissenschaft ist (Schweighauser 2014). Gleichzeitig gerät mit dieser Enthierarchisierung der Diskurse die Frage der Poetizität, der Spezifizität literarischer Sprache, weitgehend in Vergessenheit und damit auch die allgemeinere Frage, wie die gesellschaftlichen Funktionen unterschiedlicher Textgattungen durch ihre unterschiedlichen Formen bedingt sind. Wenn Jane Tompkins, die den einflussreichen Begriff der cultural work prägte, schreibt, dass literarische Texte „der Gesellschaft die Möglichkeit geben, über sich selbst nachzudenken, dass sie gewisse Aspekte einer gesellschaftlichen Wirklichkeit, die AutorInnen und LeserInnen teilen, definieren, dass sie gesellschaftliche Konflikte inszenieren und Lösungen vorschlagen“ (Tompkins 1985, 200; Übersetzung Ph. Sch.), dann schmelzen die Unterschiede zwischen politischen, erzieherischen und literarischen Texten dahin. Während das strukturalistische Projekt eines Jakobson noch untrennbar mit der Frage nach der Alterität literarischer Kommunikation verbunden war, verschiebt sich das Interesse einer auf einem poststrukturalistischen Textverständnis aufbauen- 
den historischen Literaturwissenschaft weg von der Poetizität und hin zur Poetik der Selbstinszenierung, zur performativen Kraft jeglichen Sprachgebrauchs und (bei Greenblatt) zur poetischen Verfasstheit sämtlicher Diskurse. Damit ist viel gewonnen, geht aber auch etwas Wichtiges weitgehend verloren, nämlich die Einsicht, dass unterschiedliche Textgattungen deswegen unterschiedliche kulturelle Arbeit verrichten, weil ihre Formen divergieren.

Mit dem Aufkommen des New Historicism wird gleichzeitig auch eine Grenze des Textparadigmas in der Literatur- und Kulturwissenschaft sichtbar. Ausgehend von einer poststrukturalistischen Konzeptualisierung von Text, situieren New Historicists die Literatur sehr viel stärker in ihren historischen, materiellen und politischen Kontexten, als es ein Bestehen darauf, dass alles Text sei, zulässt. Der New Historicism ist zugleich als Verwindung und Überwindung (post-)strukturalistischer Doxa angelegt und für einige seiner Vertreter ist der Dekonstruktivismus letztlich wenig mehr als ein ,New New Criticism‘: formalistisch, apolitisch und ahistorisch (Arac 1985, 346; Lentricchia 1980, 169). Weitere Bewegungen weg von einem rein textuellen Verständnis von Kultur fanden im Rahmen dessen statt, was Gottfried Boehm die ,ikonische Wende' und W. J. T. Mitchell den pictorial turn nennt (Boehm 2001; Boehm 2010; Mitchell 1992, Mitchell 1994). Boehm und Mitchell erinnern uns daran, dass Bilder eine spezifische Form und Kraft haben, welche die Ausweitung des Textbegriffs in der Nachfolge des linguistic turn - in dessen Rahmen obenstehende Überlegungen anzusiedeln sind - zu verdecken droht (Rorty 1967). Schließlich seien auch Hans Ulrich Gumbrechts und Karl Ludwig Pfeiffers gemeinsame Arbeiten und die Medienarchäologie Friedrich Kittlers genannt, welche dem hermeneutischen Projekt eine Absage erteilen und stattdessen den Blick auf die Materialität von Texten und die sie determinierenden Aufschreibesysteme sowie die sie produzierenden Schreibwerkzeuge richten. Im angelsächsischen Bereich wurden letztere Arbeiten in jüngster Zeit auch im Rahmen des breiter angelegten Projekts des surface reading rezipiert, welches statt dichter Beschreibung, close reading oder deep reading (Birkerts 1994) eine Oberflächenlektüre propagiert, die unter anderem die Materialität der Kommunikation in den Blick nimmt (Best und Marcus 2009). Damit seien zum Schluss drei neuere Zugänge innerhalb der Literatur-, Kultur- und Medienwissenschaft genannt, welche zugleich auf dem (post-)strukturalistischen Textparadigma aufbauen und dessen uneingeschränkten Geltungsanspruch bestreiten.

Ich danke A. Elisabeth Reichel und Ridvan Askin für Ihre wertvollen inhaltlichen Anregungen und Rahel Ackermann Hui für ihre sorgfältige Einrichtung des Manuskripts. 


\section{Weiterführende Literatur}

Bachmann-Medick, Doris (Hg.) (2004). Kultur als Text. Die anthropologische Wende in der Literaturwissenschaft. Frankfurt a. M.

Culler, Jonathan (1975). Structuralist Poetics. Structuralism, Linguistics, and the Study of Literature. Ithaca, NY.

Culler, Jonathan (1982). On Deconstruction. Theory and Criticism after Structuralism. Ithaca, NY. Rorty, Richard (1967). The Linguistic Turn. Recent Essays in Philosophical Method. Chicago.

Veeser, H. Aram (1989). The New Historicism. London. 